\title{
Developmental profiling of ASD-related shank3 transcripts and their differential regulation by valproic acid in zebrafish
}

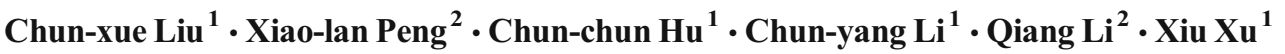

Received: 17 December 2015 / Accepted: 15 August 2016/Published online: 26 August 2016

(C) The Author(s) 2016. This article is published with open access at Springerlink.com

\begin{abstract}
SHANK3 is a scaffolding protein that binds to various synaptic proteins at the postsynaptic density (PSD) of excitatory glutamatergic synapses. SHANK3 is not only strongly implicated in autism spectrum disorders (ASD) but also plays a critical role in human Phelan-McDermid syndrome (22q13.3 deletion syndrome). Accumulated experimental evidence demonstrates that the zebrafish model system is useful for studying the functions of ASD-related gene during early development. However, many basic features of shank3 transcript expression in zebrafish remain poorly understood. Here, we investigated temporal, spatial, and isoform-specific expression patterns of shank3 during zebrafish development on the basis of previous researches and the differential effects of each shank 3 transcript expression after exposure to valproic acid (VPA), an ASD-associated drug. At first, we observed that both shank3a and shank3b were barely expressed at very early ages (before $24 \mathrm{~h}$ postfertilization (hpf)), whereas their expression levels were increased and mainly enriched in the nervous system after
\end{abstract}

Communicated by Matthias Hammerschmidt

Electronic supplementary material The online version of this article (doi:10.1007/s00427-016-0561-4) contains supplementary material, which is available to authorized users.

Qiang Li

liq@fudan.edu.cn

$\triangle \mathrm{Xiu} X \mathrm{Xu}$

xuxiu@shmu.edu.cn

1 Division of Child Health Care, Children's Hospital of Fudan University, 399 Wanyuan Road, Shanghai 201102, China

2 Center for Translational Medicine, Institute of Pediatrics, Shanghai Key Laboratory of Birth Defect, Children's Hospital of Fudan University, 399 Wanyuan Road, Shanghai 201102, China
24 hpf. Secondly, all of the six shank3 transcripts gradually increased during the first $7 \mathrm{hpf}$ and then decreased. Subsequently, they exhibited a second increasing peak between 1 month post-fertilization (mpf) and adulthood. Thirdly, VPA treatment affected the isoform-specific expression of zebrafish shank3. In particular, the mRNA expression levels of those isoforms that contain a SAM domain were significantly increased, whereas the mRNA expression level of those which contained an ANK domain but without a SAM domain was decreased. To conclude, our findings support the molecular diversity of shank 3 in zebrafish and provide a molecular framework to understand the isoform-specific function of shank 3 in zebrafish.

Keywords Autism spectrum disorder - Zebrafish .

Valproic acid · shank3 · Transcript .

Developmental expression · ANK and SAM domains

\section{Introduction}

Synaptogenesis is a key process that occurs during brain circuitry development, and inappropriate synapse formation or structure is thought to underlie a variety of neurodevelopmental disorders (van Spronsen and Hoogenraad, 2010; Guo et al., 2015). SHANK3 [SH3 and multiple ankyrin (ANK) repeat domain 3], as a member of the highly conserved SHANK/ProSAP family, is a major scaffolding protein that is enriched at the PSD of excitatory synapses (Grabrucker et al., 2011; Verpelli et al., 2011; Boccuto et al., 2013). Accumulating human genetic evidence supports a strong causal relationship between molecular defects in the SHANK3 gene and ASD, which is a heritable, debilitating neurodevelopmental disorder (Grzadzinski et al., 2013; McGuinness and Johnson, 2013; Wang et al., 2014). Moreover, recent studies using neuronal cell cultures (Verpelli et al., 2011; 
Durand et al., 2012) and mouse models (Peca et al., 2011; Yang et al., 2012; Jiang and Ehlers, 2013) have demonstrated critical roles for SHANK3 in synaptic function, social interaction and social communication, thus providing a functional link between SHANK3 deficiency and ASD behavioral features (Boccuto et al., 2013).

Recently, much experimental evidence has demonstrated that zebrafish (Danio rerio) is a valuable model organism to model human brain disorders, normal and pathological social phenotypes and other ASD-like symptoms. In particular, zebrafish can be used to explore the genetic or pharmacological modulation of those diseases (Gerlai, 2010; Kalueff et al., 2013; Stewart et al., 2013; Stewart et al., 2014), making zebrafish a strong potential model organism for studying ASD gene function early in development (Pather and Gerlai, 2009; Gerlai, 2010; Gerlai, 2011; Stewart et al., 2013).

SHANK3 appears only once in the human genome. Nevertheless, this gene is duplicated in zebrafish, appearing as shank3a (chromosome 18) and shank3b (chromosome 4) (Kozol et al., 2015). Further examination of shank3a and shank $3 b$, as well as their different variants, will make a valuable contribution to the study of the function of human SHANK3.

Five intragenic promoters (Wang et al., 2011; Jiang and Ehlers, 2013; Zhu et al., 2014) and extensive alternative splice variants have been identified in mouse and human SHANK3 (Lim et al., 1999; Wilson et al., 2003; Durand et al., 2007), which all contribute to the diversity of Shank 3 transcripts. Moreover, Shank3 transcripts exhibit dynamic spatiotemporal domains during the developmental stage when neural segment regions are molded (Wang et al., 2014). The complexity of Shank3 transcriptional regulation has been reported in mouse (Jiang and Ehlers, 2013; Wang et al., 2014; Zhu et al., 2014). Therefore, the clinical heterogeneity in the disorders caused by SHANK3 mutations may be due to the differential effects of the variable locations of mutations within the coding exon of each SHANK3 isoform.

A recent study has determined the developmental expression patterns of shank $3 a$ and shank $3 b$ in zebrafish, ranging in the age from 2 to $120 \mathrm{hpf}$, and two transcripts of shank 3 gene have been verified and characterized (Kozol et al., 2015). However, six shank3 transcripts exist according to the Ensembl database (Zv9). It is urgent to further study the developmental expression of all shank 3 transcripts in zebrafish during more developmental stages, so as to improve the expression profile characteristics. Examining and analyzing the profiles of these shank 3 transcripts may provide a foundation for the further establishment of a transgenic zebrafish model of shank3.

Epigenetic modifications, resulting from enzymes such as histone deacetylase (HDAC), have been shown to regulate the expression and alternative splicing of neuronal genes (Schor et al., 2009; Fischer et al., 2010). VPA is a typical example of
HDAC inhibitor that was recently found to have teratogenic (interfering with early development) and neuropsychiatric side effects related to in utero exposure (Jacob et al., 2014). Moreover, fetal VPA exposure has been associated with a 3- to 46-fold increased risk of ASD (Dufour-Rainfray et al., 2010; Bromley et al., 2013; Christensen et al., 2013; Wood, 2014). Therefore, in this study, VPA was applied to investigate the alterations of the expression levels of the different shank3 transcripts and to dissect the distinct functions of each conserved domain.

In general, examination of the transcriptional and developmental expression patterns as well as the differential effects on zebrafish shank 3 transcripts in response to VPA treatment is crucial to establish a shank3-knockout zebrafish model for elucidating the functions of each shank 3 isoform and exploring the mechanisms underlying ASD clinical heterogeneity.

\section{Materials and methods}

\section{Fish and embryo maintenance}

Wild-type zebrafish (Tu) were raised and maintained under standard laboratory conditions at $28.5^{\circ} \mathrm{C}$ in "system water" under a $14 \mathrm{~h}$ light $/ 10 \mathrm{~h}$ dark cycle (Kalueff et al., 2014). Freshly fertilized eggs were collected from multiple breeding tanks containing 25 females and 25 males.

\section{Evolutionary comparison and verification of six zebrafish shank3 transcripts}

The zebrafish shank3 nucleotide sequence, exon-intron structure, amino acid sequence, different transcripts and conserved domains were analyzed using the NCBI gene database (http://www.ncbi.nlm.nih.gov/gene/) and zebrafish whole genome sequence project database (http://www.ensembl. org/Danio_rerio). Protein sequences belonging to the shank3 family were identified using the NCBI BLAST program and confirmed by best reciprocal BLAST.

In particular, since the $5^{\prime}$ open reading frame (ORF) of shank3a-1 was incomplete, PCR amplification was then performed on cDNA from 3-month-old zebrafish with primers designed based on conservative regions of the predicted ORF sequence (forward: 5'-GAGAGTGTATGCATGAGGGAGGCAC$3^{\prime}$ and reverse: $5^{\prime}$-GGTCTGGGTCTCCATATACAGTACCCC3', Supplementary Material, Fig. S1). The PCR conditions were as follows: $95^{\circ} \mathrm{C}$ for $2 \mathrm{~min}, 35$ cycles of $95^{\circ} \mathrm{C}$ for $30 \mathrm{~s}, 56^{\circ} \mathrm{C}$ for $30 \mathrm{~s}, 72^{\circ} \mathrm{C}$ for $4 \mathrm{~min} 10 \mathrm{~s}$, and a final extension at $72^{\circ} \mathrm{C}$ for $5 \mathrm{~min}$. The PCR products were then cloned into a pGEM-T Easy vector (Promega) and sequenced.

To analyze SHANK3 gene evolution and conservation, multiple alignments were performed and phylogenetic trees constructed using MEGA 6. The alignments were imported 
into the BoxShade Server (http://www.ch.embnet. org/software/BOX form.html) to identify regions of high local similarity.

Based on the sequences in the Ensembl database, transcript-specific oligonucleotide primers and amplicons were generated (Supplementary Material, Fig. S1 and Table S1). cDNA from 3-month-old zebrafish was used as a template for PCR, as noted above each $2 \%$ agarose gel in Fig. 2b.

\section{Spatial expression pattern of larval zebrafish shank $3 a$ and shank $3 b$ detected by whole-mount in situ hybridization}

Larvae were dechorionated and placed in a mixture of fish facility system water and $0.003 \%$ phenylthiourea at $24 \mathrm{hpf}$. Embryos were fixed and processed for WISH. Table S2 in the Supplementary Material lists the WISH primers that were used to amplify and synthesize RNA probes. The PCR products were then cloned into a pGEM-T Easy vector. Sense and antisense RNA probes were individually transcribed using linearized constructs with T7 or Sp6 polymerase (Ambion, USA) in the presence of digoxigenin (DIG, Roche, Germany)-labeled UTP using a DIG-RNA Labeling Kit (Roche, Germany). The resulting DIG-labeled antisense probes were used to label the shank3a and shank3b genes respectively, and the sense probes were used as negative controls. WISH (approximately 40 eggs/clutch) was performed as described previously (Thisse and Thisse, 2008) using 5-nitroblue tetrazoliumchloride and bromo-4-chloro-3'-indolyl phosphate p-toluidine salt (NBT/BCIP, Vector Laboratories) as substrates (Thisse and Thisse, 2008). After WISH, the embryos were mounted in $4 \%$ methylcellulose, and images were captured using a Leica 205C microscope.

\section{VPA treatments and phenotypic assessments}

Embryos were dechorionated prior to drug treatment. Zebrafish embryos were exposed to a final concentration of 0.5 or $1.0 \mathrm{mM}$ VPA (P4543-10G, Sigma, USA) or a control solution ( $0.3 \mathrm{X}$ Danieau's solution), which was administered continuously from $24 \mathrm{hpf}$ until $48 \mathrm{hpf}$.

Embryos exposed to VPA or the control solution were observed for morphological abnormalities at $48 \mathrm{hpf}$. The embryos were scored individually for several phenotypes, including developmental retardation, pericardial effusion, spinal curvature and reduced pigmentation. Each treatment consisted of three biological replicates with 70 embryos per replicate. During drug treatment, VPA-exposed and control zebrafish embryos were observed under a microscope for any morphologic abnormalities. The data were presented as the percentages of embryos with each defect, and the results were calculated by dividing the total number of embryos with the deformity by the total number of embryos in each replicate. Samples were collected at $48 \mathrm{hpf}$ and stored at $-80^{\circ} \mathrm{C}$ until RNA isolation.

\section{Quantitative real-time PCR}

Approximately 20 zebrafish embryos at 1, 3, 5, 7, 10, 13, 15, 30, and 60 days post-fertilization (dpf) were used for each condition, and each experiment was performed in triplicate. Total RNA was extracted from the embryos using TRIzol reagent (Ambion, USA). Extracted RNA was treated with DNase (Life Technologies, USA) according to the manufacturer's protocol. Whole RNA samples were run on $2 \%$ agarose gels to determine RNA integrity. Reverse transcription was performed with a PrimeScript $^{\mathrm{TM}}$ RT Reagent Kit (TaKaRa, Japan). q-PCR was performed using a LightCycler ${ }^{\circledR} 480$ Instrument (Roche, Germany) and SuperReal PreMix Plus (TIANGEN, China) according to the manufacturer's recommendations. The q-PCR conditions were as follows: an initial cycle of $95{ }^{\circ} \mathrm{C}$ for $15 \mathrm{~min}$; 40 cycles of $95{ }^{\circ} \mathrm{C}$ for $30 \mathrm{~s}, 60^{\circ} \mathrm{C}$ for $30 \mathrm{~s}$, and $72{ }^{\circ} \mathrm{C}$ for $15 \mathrm{~s}$; and a final cycle of $95^{\circ} \mathrm{C}$ for $1 \mathrm{~min}$; followed by $40^{\circ} \mathrm{C}$ for $1 \mathrm{~min}$, $65^{\circ} \mathrm{C}$ for $1 \mathrm{~min}, 95^{\circ} \mathrm{C}$ for $1 \mathrm{~min}$; and finally $40^{\circ} \mathrm{C}$ for $1 \mathrm{~min}$. As an internal control, a 102-bp sequence of $\beta$-actin cDNA was amplified by q-PCR using two specific primers (Supplementary Material, Table S1). The primers used in this study were designed using Primer3 and by running BLAT (https://genome.ucsc. edu/cgi-bin/hgBlat) against the zebrafish genome to confirm gene specificity and they are listed in Table S1 in the Supplementary Material (Rozen and Skaletsky, 2000). The sequences of the newly identified splice variants were annotated and deposited into the Ensembl database.

\section{Results}

shank3 is a conserved gene in the vertebrate lineage that possesses multiple transcripts

The $5^{\prime}$ incomplete fragment of shank3a-1 (Supplementary Material, Fig. S1) was isolated by PCR using specific primers designed to acquire the full length of the ORF. As verified by PCR and Sanger sequence analysis, shank $3 a-1$ was termed as the completed-length cDNA and has been studied in depth in our study.

According to the NCBI BLAST Alignment results (http://blast.ncbi.nlm.nih.gov/), the longest shank3a (shank3a-1, our study) and shank3b (shank3b-1) protein sequences of Danio rerio (Fig. 1a) exhibit a high level of amino acid identity (55 and $59 \%$, respectively) with the corresponding Homo sapiens SHANK3 protein (NP 277052.1). In particular, the four typical conserved domains (ANK, SH3, PDZ, and SAM domain) of shank3 exhibit at least $80 \%$ amino acid identity 
Fig. 1 Multiple transcripts of shank3 in zebrafish. a The structures of six zebrafish shank3 transcripts are shown. Yellow rectangles indicate the $5^{\prime}$ and $3^{\prime}$ UTRs. Notably, the SHANK3 gene is duplicated in zebrafish, appearing as shank3b (chromosome 4) and shank3a (chromosome 18). b Extensive predicted protein isoforms of zebrafish shank3. The protein domains are shown and aligned to corresponding exons (ANK ankyrin repeat domain, $\mathrm{SH} 3 \mathrm{Src}$ homology 3 domain, PDZ PSD95/discs large/ZO-1 domain, SAM sterile alpha motif domain a

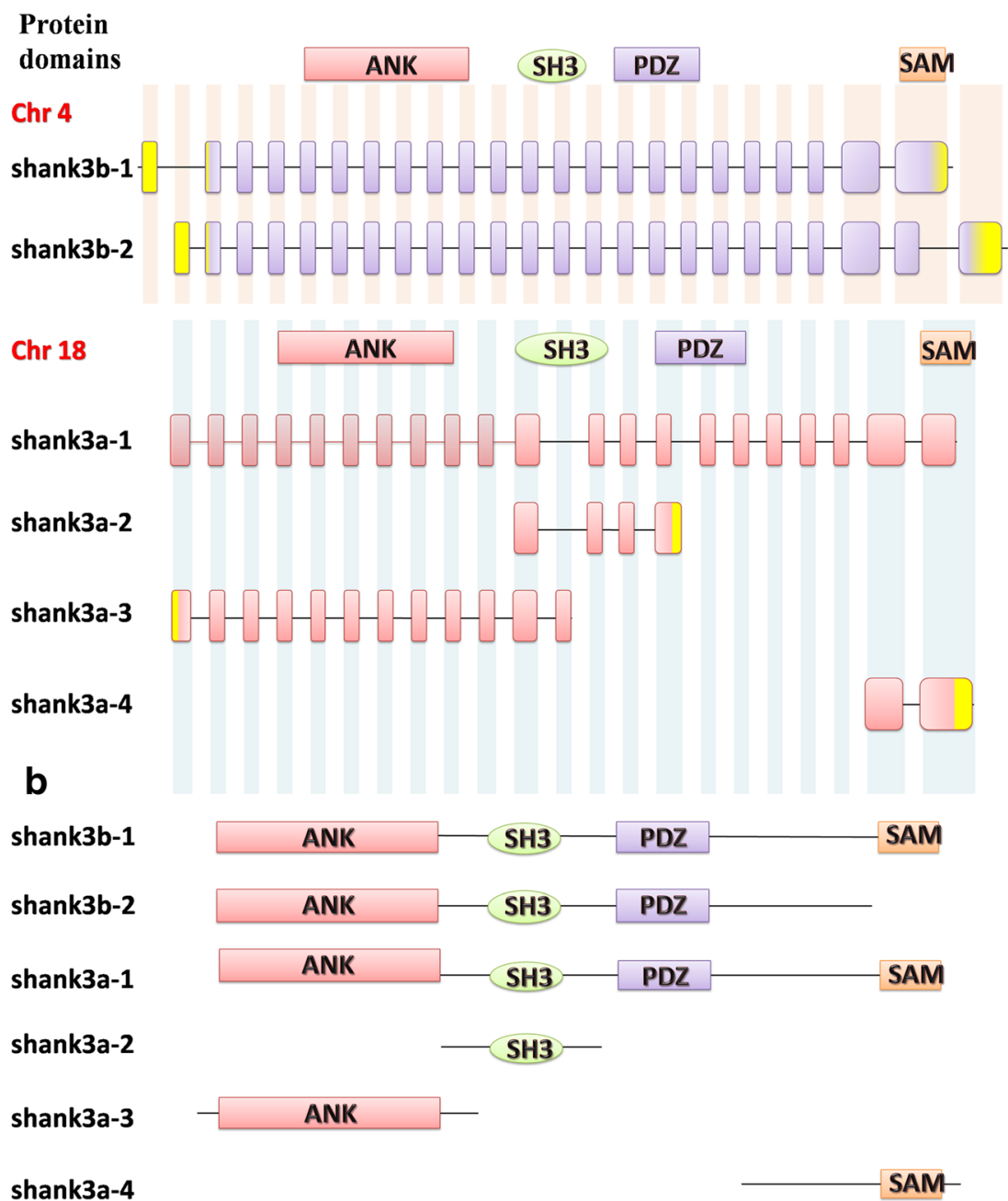

(Supplementary Material, Fig. S2). These findings indicate that human SHANK3 is highly conserved in zebrafish and that zebrafish might be a strong potential model for studying human SHANK3.

To further examine the orthologs between zebrafish shank3 and other species, we performed phylogenetic analysis to compare shank 3 of Danio rerio with that of Homo sapiens and 11 other species by constructing a phylogenetic tree (Fig. 2a). The obtained numbers represented evolutionary relationships, with larger numbers indicating greater genetic differences. In vertebrate animals, the genetic distance was not greater than " 0.3 "; however, in invertebrates (such as Bactrocera cucurbitae), the distance was as high as "1.5" (Fig. 2a). Therefore, this phylogenetic analysis revealed a highly conserved pattern in mammals, rodents, and other vertebrates, implying that shank3 is unique to the vertebrate lineage.

However, the alternative splicing of shank 3 in zebrafish has not been fully characterized (Gauthier et al., 2010).
Based on the sequences in the Ensembl database, each transcript of zebrafish shank 3 has some unique sequences that are either in the exon or intron of other transcripts. We were able to generate transcript-specific oligonucleotide primers and amplicons to confirm the existence of shank3 splice variants (Supplementary Material, Fig. S1). The amplification results confirmed that the key sequences of the six zebrafish shank3 transcripts did exist (Fig. 2b).

\section{Spatial and temporal expressions of shank 3 in zebrafish}

To determine the spatial and temporal expression patterns of shank3a and shank3b in zebrafish, we chose the longest zebrafish shank3a transcript (shank3a-1) and shank3b transcript (shank3b-1) to perform the whole mount in situ hybridization at 2, 10, 14, 24, 48, and $72 \mathrm{hpf}$. We found that both of them exhibited a specific pattern of concentrated expression in the zebrafish larval brain. At early ages (before $24 \mathrm{hpf}$, 
a
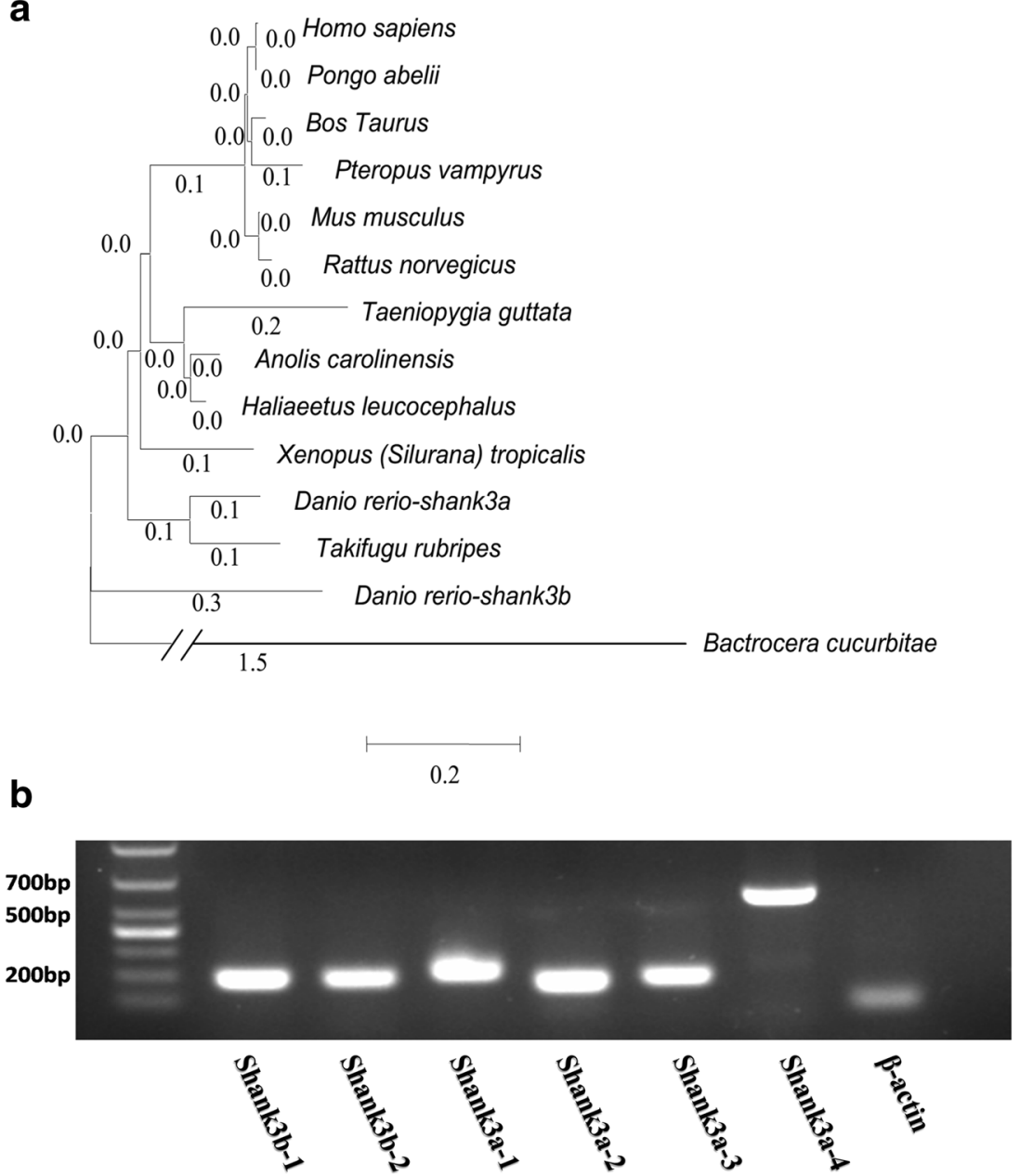

Fig. 2 Phylogenetic tree of evolutionary relationship of SHANK3 proteins. a Diverse animal phyla were identified by a best reciprocal BLAST search with human SHANK3 and are mapped onto a phylogenetic tree. All of the sequences are available from the NCBI protein database with the exception of zebrafish shank3a, which was obtained from the Ensemble protein database and our study. Homo sapiens (NP_277052.1), Pongo abelii (XP_009232827.1), Rattus norvegicus (NP_067708.1), Mus musculus (NP_067398.2), Bos Taurus (XP_010804153.1), Taeniopygia guttata (XP_012432256.1), Xenopus (Silurana) tropicalis (XP_002941881.2), Danio rerio shank3b (XP 001919745.1), Bactrocera cucurbitae (XP 011184638.1), Pteropus vampyrus (XP_011382480.1), Takifugu rubripes (XP_003967184.1),
Anolis carolinensis (XP 008121382.1), Haliaeetus leucocephalus (XP 010564365.1), and Danio rerio shank3a (ENSDART00000139505 and our study). The tree was constructed using the neighbor-joining methods as implemented in the MEGA 6 package. Clade robustness was measured by the bootstrap method with 1000 replicates. The lengths of the lines are proportional to the evolutionary distances from branching points. b Transcript-specific oligonucleotide primers and amplicons were generated to confirm the existence of the key fragment of six predicted transcripts of shank3 in the Ensembl database. cDNA from 3-month-old zebrafish was used as a template for PCR, as noted above each $2 \%$ agarose gel. The housekeeping gene $\beta$-actin was used as an internal reference
Fig. 3a-c, j-1), both shank3a and shank3b were barely expressed, whereas their expression levels increased after $24 \mathrm{hpf}$ (Fig. $3 \mathrm{~d}-\mathrm{i}, \mathrm{m}-\mathrm{r}$ ) and tended to increase throughout development in zebrafish larvae. In addition, the expression levels of shank $3 a$ and shank $3 b$ on $24 \mathrm{hpf}$ were similar, whereas on 48 and $72 \mathrm{hpf}$, shank3a displayed a higher expression level than shank $3 b$. Moreover, the expressions of both of these isoforms were mainly concentrated in the nervous system of zebrafish larvae, particularly in the anterior-ventral forebrain.
To further distinguish the different developmental profiles of each shank3 isoform, isoform-specific qPCR was performed using whole brains from wild-type zebrafish on nine different developmental stages (Fig. 4g). Remarkably, the expression of all zebrafish shank3 transcripts gradually increased over the first $7 \mathrm{dpf}$ (Fig. 4g, a-f). The most obvious increasing trend in shank3 expression was observed from 3 to $7 \mathrm{dpf}$, correlating with the WISH expression pattern and the time frame for synaptogenesis. A peak in shank3 expression was observed on approximately $7 \mathrm{dpf}$. 
Fig. 3 Analysis of regionspecific expression of shank $3 a$ and shank $3 b$ in wild-type zebrafish by WISH. a-i WISH analysis of the level and pattern of shank $3 a$ and shank $3 b$ expressions at $2,10,14,24,48$, and $72 \mathrm{hpf}$ were showed. The black arrow heads in $\mathbf{d}-\mathbf{i}$ and $\mathbf{m}-\mathbf{r}$ show the expression signals in the zebrafish larva brain. Obviously, the expression of both of these isoforms was mainly concentrated in the nervous system of zebrafish larvae, particularly in the anteriorventral forebrain shank3b

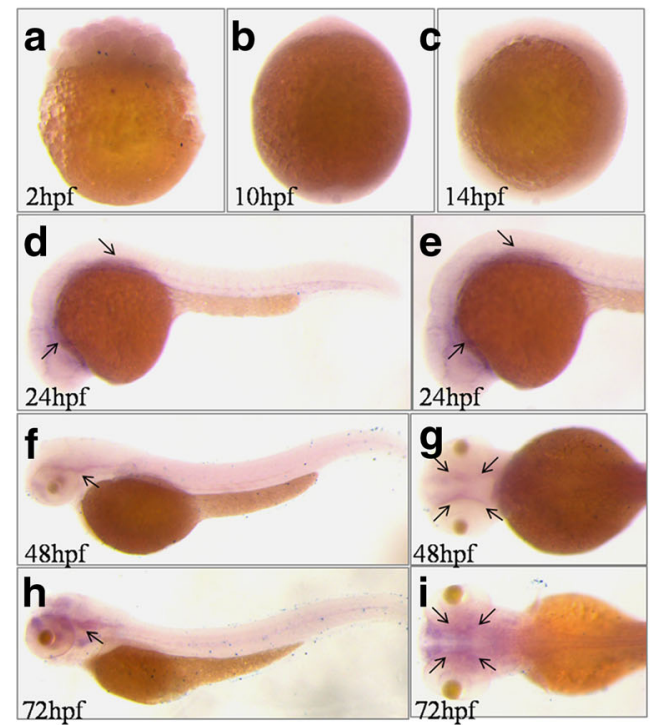

shank3a

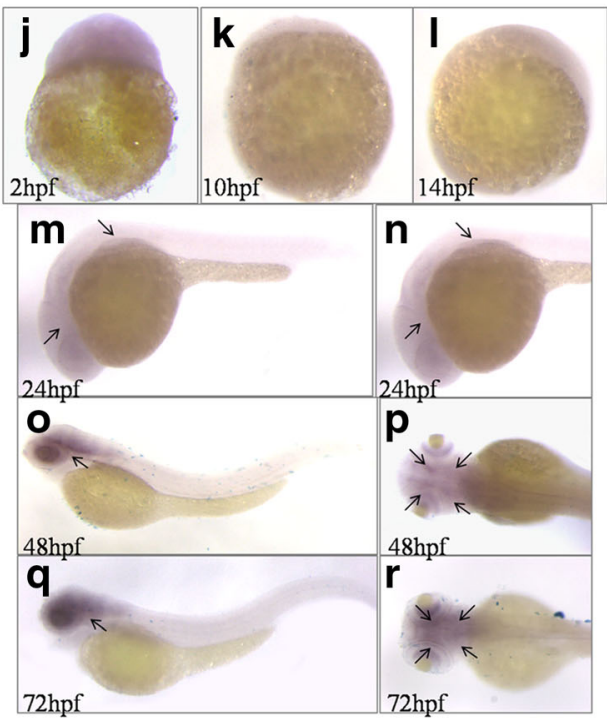

Notably, all of the shank3 transcripts displayed a gradual decrease in expression after $7 \mathrm{dpf}$, and their expression levels, with the exception of that of shank3b-2 (Fig. 4b), decreased by at least $50 \%$ compared with the peak point by $15 \mathrm{dpf}$. In addition, these transcripts displayed a second increasing peak between $1 \mathrm{mpf}$ and adulthood. The expression levels of shank3b-1 (Fig. 4a), shank3a-1 (Fig. 4c) and shank3a-3 (Fig. 4e) markedly increased from 5 to $7 \mathrm{dpf}$. In contrast, the shank3b-2 (Fig. 4b) and shank3a-4 (Fig. 4f) expression levels were relatively stable before adulthood.

\section{Isoform-specific expression pattern of shank3 during development}

To perform an in-depth analysis of the differential expression of each isoform at the same age stage, the isoform-specific expression of zebrafish shank3 was then examined (Fig. 5). The expression levels of shank3b transcripts (shank3b-1 and shank3b-2) were low throughout development; whereas all shank3a transcripts (shank3a-1, shank3a-2, shank3a-3 and shank3a4) displayed relatively higher expression levels. On $1 \mathrm{dpf}$, all of the transcripts showed relatively lower expression levels (Fig. 5a). However, during the period from 3 to $10 \mathrm{dpf}$ (Fig. 5b-e), the expression levels of all shank3a transcripts were obviously higher than that of shank3b, which is consistent with the WISH results (see above). Moreover, the difference between shank3b1 and shank3b-2 was not significant from 1 to $5 \mathrm{dpf}$ (Fig. 5a-c), whereas after $7 \mathrm{dpf}$, the expression of shank3b-1 was higher than that of shank3b-2.
The ANK and SAM domains of zebrafish shank3 may display the opposite effect following treatment with VPA

To examine whether different shank3 transcripts were subject to HDAC, zebrafish embryos were treated with VPA. The concentrations that caused teratogenic effects, as detailed in the literature, varied among studies but generally ranged from 0.1 to $3.0 \mathrm{mM}$ (Li et al., 2009; Selderslaghs et al., 2009; Brannen et al., 2010; Terbach et al., 2011; Cowden et al., 2012; Teixido et al., 2013). Thus, an initial dose-response experiment was conducted to determine the effects of two different VPA concentrations, 1 and $0.5 \mathrm{mM}$, on zebrafish embryos (Fig. 6). The VPA-treated zebrafish embryos displayed numerous morphological abnormalities, and those exposed to a higher concentration displayed more deformities. Most embryos exposed to $1 \mathrm{mM}$ VPA exhibited phenotypic abnormalities, including developmental retardation (100\%, $70 / 70)$, reduced pigmentation $(100 \%, 70 / 70)$, spinal curvature (43\%, 30/70) and pericardial effusion (64\%, 45/70, Supplementary Material, Figs. S3A and B). Additionally, embryos that exposed to $0.5 \mathrm{mM}$ VPA displayed relatively fewer defects, such as developmental retardation (100\%, 70/70), reduced pigmentation ( $83 \%, 58 / 70)$, spinal curvature $(20 \%$, $14 / 70)$ and pericardial effusion (40\%, 28/70, Supplementary Material, Figs. S3C and D).

Notably, the VPA treatment exhibited varying effects on different shank3 transcripts. In the zebrafish embryos treated with $1 \mathrm{mM}$ VPA, the expressions of all transcripts containing the SAM domain (shank3b-1, shank3a-1and shank3a-4, Fig. 1b) were significantly increased (Fig. 6a), whereas of the expressions of shank3b-2 and shank3a-3 (Fig. 1b), which contained an ANK domain but without the SAM domain, were remarkably decreased (Fig. 6a). The opposite direction 
Fig. 4 Differential expression of zebrafish shank3 transcripts throughout development. a-g The expression of six shank 3 transcripts of zebrafish from 1 day to 3 months post-fertilization was determined by q-PCR. The crossing point $(\mathrm{CP})$ values of each transcript were normalized to that of a housekeeping gene, $\beta$-actin. The error bars denote the standard deviations from three RNA sample replicates for each developmental time point. All data are shown as the means \pm SEM a

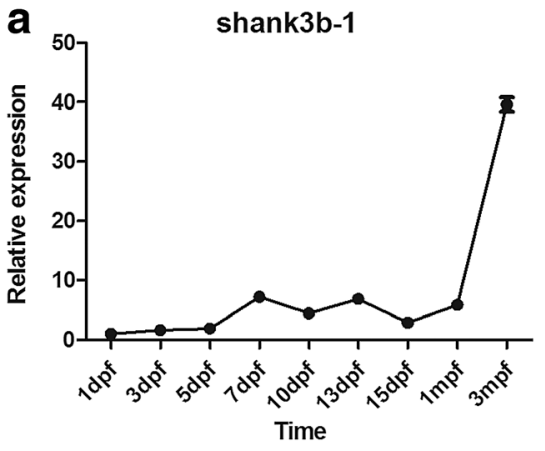

C
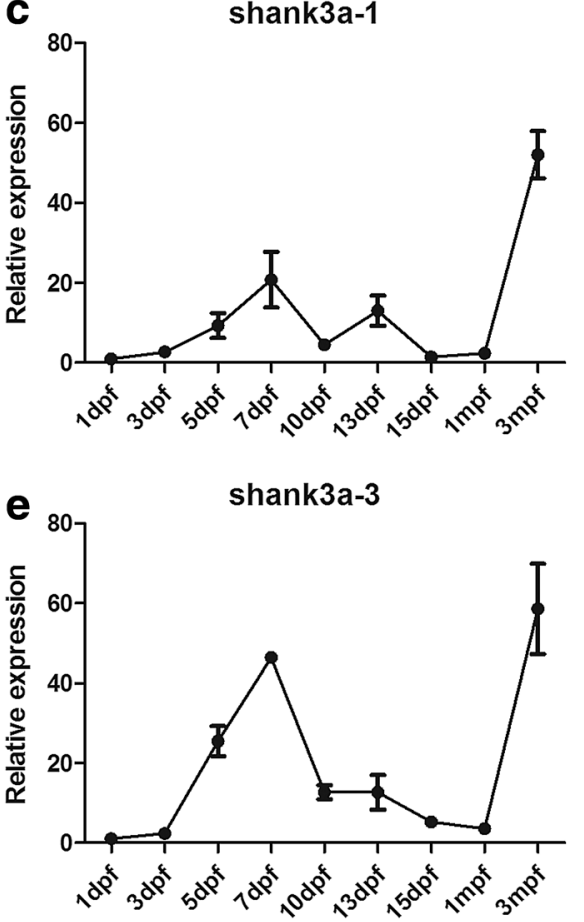

b ${ }^{30} \quad$ shank3b-2

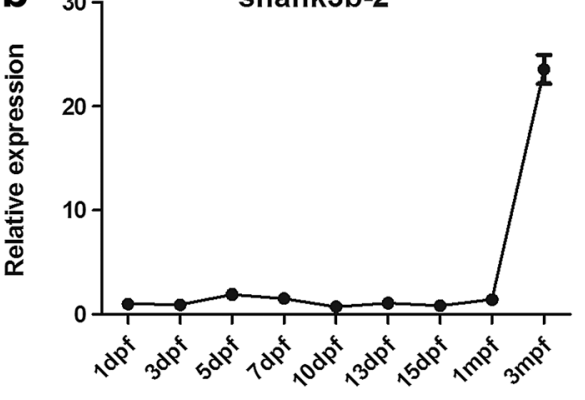

d

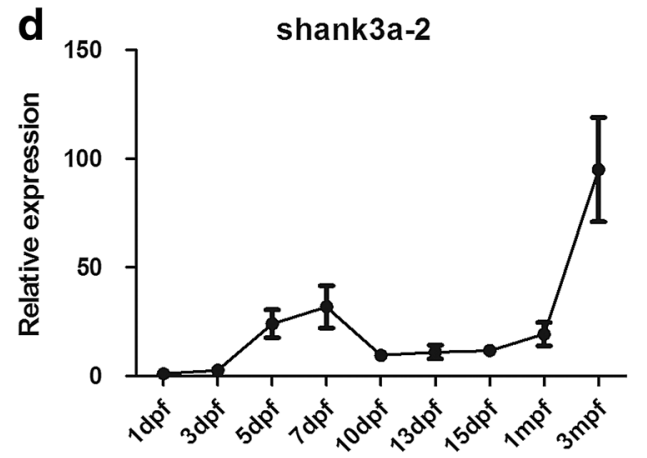

$\mathbf{f}$

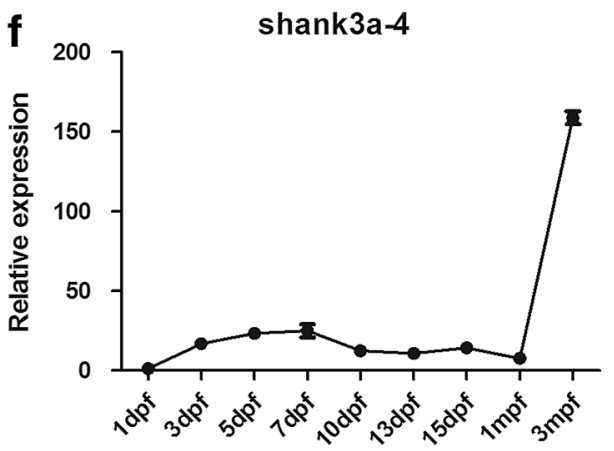

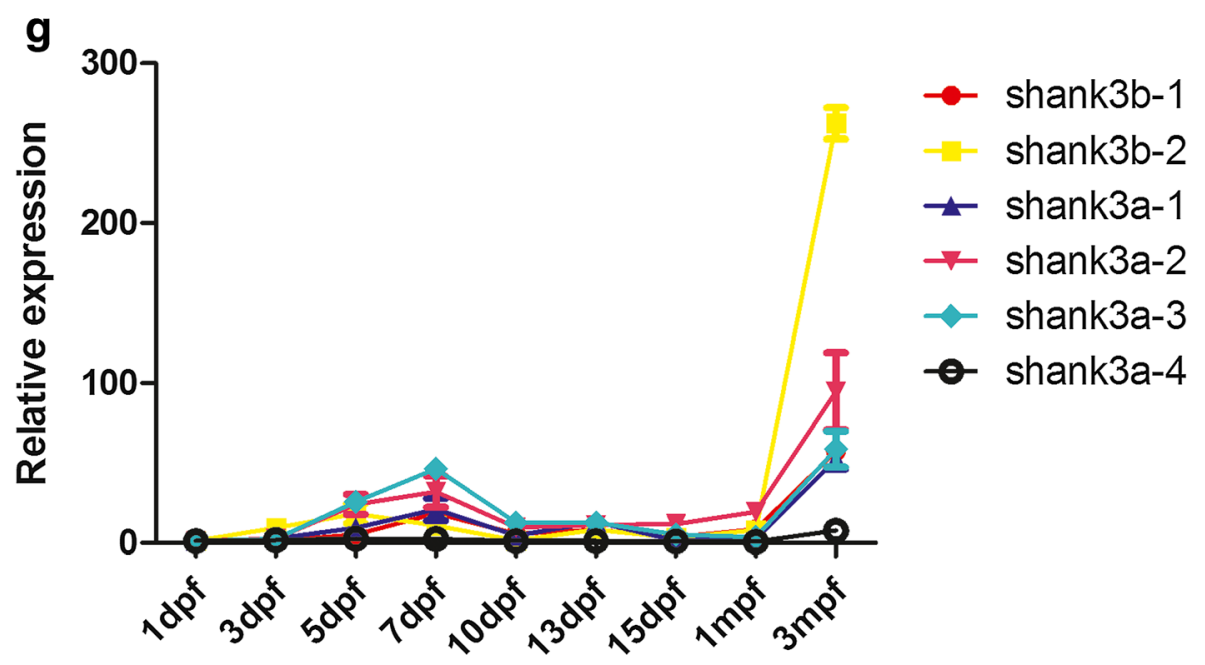

was also replicated in lower VPA concentrations $(0.5 \mathrm{mM}$, Fig. 6b). In summary, our data suggest that the ANK and
SAM domains of shank3 are more susceptible to VPA and are crucial for the normal function of shank3. 

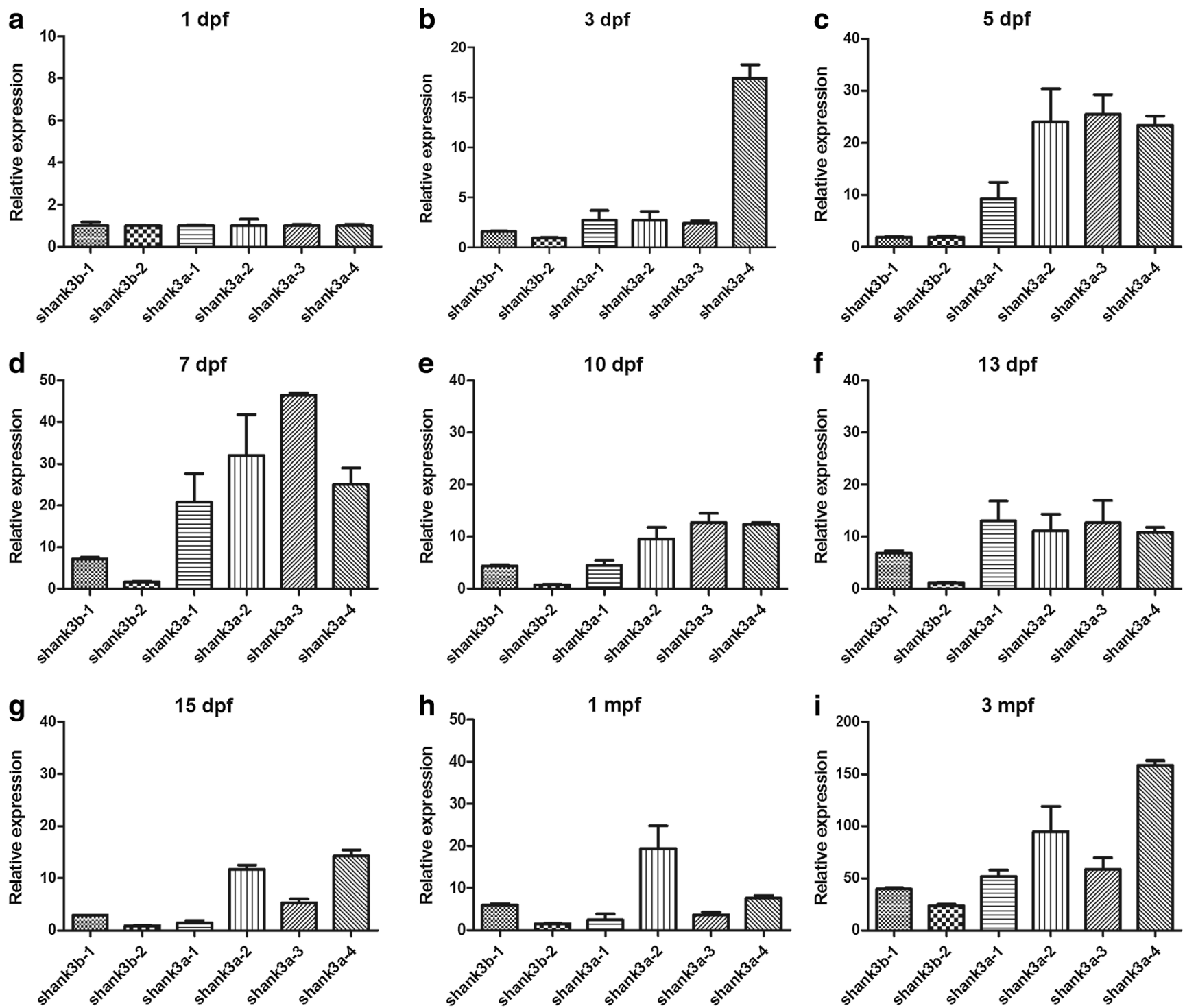

Fig. 5 Isoform-specific pattern during the same period. a-i Comparisons of the expression patterns of six different shank 3 isoforms in the zebrafish brain at the same stage are shown. The crossing point $(\mathrm{CP})$ values of each

error bars denote the standard deviations from three RNA sample replicates for each developmental time point. All data are shown as the means \pm SEM isoform were normalized to that of a housekeeping gene, $\beta$-actin. The

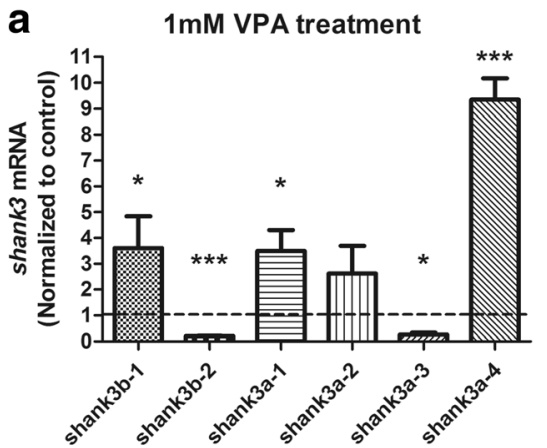

Fig. 6 Isoform-specific expression of zebrafish shank3 induced by VPA compared with the control. a, b q-PCR analysis of zebrafish shank3 transcripts at $48 \mathrm{hpf}$ after treatment with 1 or $0.5 \mathrm{mM}$ VPA for $24 \mathrm{~h}$. All data are shown as the means \pm SEM. The statistical significance values,

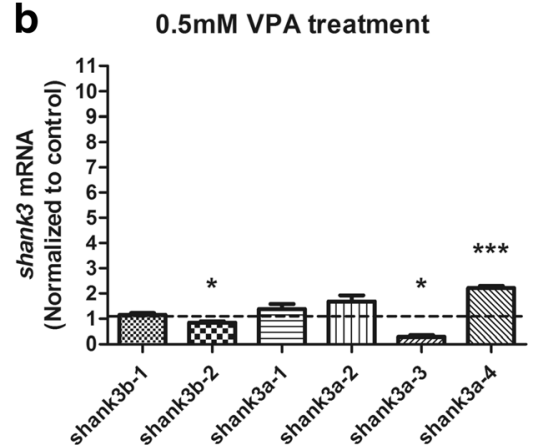

such as $* P<0.1, * * P<0.01$, and $* * * P<0.005$, of differences compared with the control group were determined through two-tailed $t$ test $(n=15$ in each group) 


\section{Discussion}

Our study demonstrated that zebrafish shank3 was unique to the vertebrate lineage (Fig. 2a) and possessed an ortholog of human SHANK3 that has retained the conserved ANK, SH3, PDZ and SAM domains (Supple mentary Material, Fig. S2). Comprehensive and systematic experiments were performed to analyze the isoformspecific expression of shank3, which displayed temporal and spatial specific patterns. In particular, VPA could regulate the expression of shank3 in zebrafish, and shank3 transcripts containing a SAM domain displayed significantly decreased expression patterns, whereas those containing an ANK domain but without the SAM domain showed the opposite trend, supporting the hypothesis that each isoform has distinct functions because of the different features of each domain.

Taken together, these findings will enhance the current knowledge of the molecular diversity in the brain and provide insights into the phenotypic heterogeneity caused by various SHANK3 defects in humans.

\section{The expression profiles of shank 3 may be linked with synaptic development}

Shank3 expression has been widely characterized in mammalian animal models (Jiang and Ehlers, 2013; Wang et al., 2014), and the spatial expression patterns of shank3a and shank3b at $48 \mathrm{hpf}$ in zebrafish have also been determined (Kozol et al., 2015). On the basis of previous research, we further validated the expression patterns of more developmental stages. The results revealed that zebrafish shank3 was mainly expressed in the nervous system, particularly in the anterior-ventral forebrain (Fig. 3d-i, m-r). Over the first 3 days, almost all shank 3 transcripts displayed a low level of expression (Fig. 4g); thereafter, a rapid increase in expression that corresponded with the time frame for synaptogenesis was observed. As a scaffolding protein, shank3 played a key role in synaptic formation, maturation, maintenance, and transmission. The increasing expression over the first several days corresponded with the rapid development of the nervous system, which indirectly reflected the increasing head circumferences in children with age. The increase in brain size over time was due to the early growth of gray matter followed by the pruning of dendrites and synaptic structures, combined with major growth of white matter as it underwent myelination (Geschwind and Levitt, 2007). Shaw et al. (Shaw et al., 2006) suggested that cognitive skills were more significantly correlated with the developmental trajectory of brain volumes over time than with static brain volume.

Our results also demonstrated that the expressions of shank3 transcripts declined afterward, which largely coincided with the data obtained from mouse studies (Wang et al., 2014). Wang et al. examined the developmental expression of five Shank3 transcripts in mouse brains with different ages and observed a peak of expression that occurred between 2 and 4 weeks. However, in our results, the first peak was observed on approximately $7 \mathrm{dpf}$ and the second peak was detected during adulthood. In addition, in mouse studies (Wang et al., 2014), two transcripts (Shank3a and Shank3b) displayed similar trends to those of shank3b-1 and shank3b-2, respectively, in our study. Interestingly, in-depth investigations into the protein structures revealed that these mouse and zebrafish transcripts share the same conserved domains. The other four shank3 transcripts (shank3a-1, shank3a-2, shank3a-3 and shank3a-4) in zebrafish differed from those in the mouse, which may reflect functional differences. Therefore, a more thorough analysis of these different transcripts would compensate for insufficiencies in mouse models.

Two recent studies have analyzed the shank3 gene in zebrafish (Gauthier et al., 2010; Kozol et al., 2015). Both studies used morpholinos to knock down the expression of shank3a and shank3b (Kozol et al., 2015) or zs3.1 and zs3.2 (Gauthier et al., 2010), systematically clarifying the functions of the two shank3 transcripts. Upon performing a BLAST search of the morpholino oligonucleotides used in their studies (Supplementary Material, Fig. S4), we found that shank3a (Kozol et al., 2015) corresponded to shank3a-1 and shank3a-3 in our study and that shank3b (Kozol et al., 2015) corresponded to shank3b-1 and shank3b-2. Thus, only some of the shank 3 transcripts were likely knocked down, whereas the others remained unaffected. Furthermore, in the study of Gauthier et al., only four transcripts (shank3b-1, shank3b-2, shank3a-1 and shank3a-2) were knocked down (Gauthier et al., 2010). A further study of the functions of the remaining transcripts is warranted. In addition, the studies of these transcripts are currently underway in our laboratory.

Parenthetically, each shank3 isoform has a unique combination of protein domains. Full-length shank3a-1and shank3b-1 contain all four domains (ANK, SH3, PDZ, and SAM), which allow for their interactions with all possible interacting proteins. Other isoforms with different combinations of functional domains interact with different subsets of synaptic proteins. For instance, the Cterminal truncated shank3a-3 (Fig. 1b) can only interact with $\alpha$ fodrin but not with mGluRs, Homer1 or other partners. Because each protein domain mediates a unique complement of proteinprotein interactions (Hayashi et al., 2009), each shank3 isoform likely has distinct functions.

Taken together, these results provide evidence to support the findings that different shank 3 isoforms have distinct functions at synapses and are associated with different clinical presentations. However, the exact mechanism regulating shank 3 transcription in zebrafish remains to be investigated in the future. 


\section{The SAM and ANK domains may be a key connection between shank3 and VPA}

The epigenetic modification of DNA by the methylation of cytosine in $\mathrm{CpG}$ dinucleotides and histone modification represented a common mechanism to regulate the gene expression (Collas, 1998). VPA is a known HDAC inhibitor that could activate genes through reprogramming of genome expression and by causing hyperacetylation of induced genes (Milutinovic et al., 2006).

In this study, we demonstrated that VPA treatment altered shank3 mRNA expression during zebrafish development. To the best of our knowledge, this is the first study evaluating the effects of VPA on shank3 transcript expression in zebrafish. Treatment with VPA during embryonic development induced a delayed development in hatching and an increased number of malformations. The toxic responses to VPA (1 and $0.5 \mathrm{mM}$ ) were dose-dependent (Terbach et al., 2011).

Notably, the expression levels of shank3b-1, shank3a-1 and shank3a-4 significantly increased after treatment with $1 \mathrm{mM}$ VPA (Fig. 6a). Further analyses of their protein structures revealed that the three transcripts all contained a SAM domain (Fig. 1b). The SAM domain was next to the $\mathrm{C}$ terminus of shank3 and these proteins bound to each other in a homomeric and heteromeric manner, indicating that shank3 proteins could multimerize in a tail-to-tail manner (Sheng and Kim, 2000).

Another interesting finding was that the expression levels of shank3b-2 and shank3a-3, which lacked the SAM domain but contained the ANK domain, were markedly decreased after exposure to both 1 and $0.5 \mathrm{mM}$ VPA (Fig. 6a). However, full length shank3a-1 and shank3b-1 isoforms that encoded all protein interaction domains exhibited the reverse effect. One possible explanation was that the SAM domain likely offsetted the increasing effect. The ANK domain associated with the actin-based cytoskeleton and was conducive to dendritic spine development (Lim et al., 1999; Jiang and Ehlers, 2013). $\alpha$-Fodrin has been shown to interact with the N-terminal ANK repeats of shank3 (Bockers et al., 2001). In addition, ANK repeats exhibited secondary structures in the form of pairs of anti-parallel $\alpha$-helices that were linked by a variety of $\beta$-hairpin motifs. However, these repeats did not prefer specific motifs, nor did they recognize consensus sequences of the target molecules (Sedgwick and Smerdon, 1999; Bockers et al., 2001).

Shank3 contains multiple domains for protein-protein interactions, but understanding of their functional significance for each domain is lacking (Sheng and Kim, 2000). Our results suggested that VPA selectively altered the expression of shank3 transcripts containing the SAM and ANK domains and implied a functional correlation for the Shank3 proteins with these domains.
The complexity of the shank3 transcript structure indicates that the point mutations, translocations, and intragenic deletions of shank3 observed in ASD patients are isoform-specific (Jiang and Ehlers, 2013). Further studies are necessary to determine the functions of each isoform in vivo and their relevance to synaptic and behavioral phenotypes. Understanding the unique function of each shank3 transcript will undoubtedly be beneficial for future drug screening.

Acknowledgments We thank all members of the Zebrafish Core of Children's Hospital of Fudan University in China. We also thank Yonghui Jiang and Xiao-ming Wang for the advice on experimental design and Ling-li Xu for the assistance of bioinformatics analysis. This study was supported by grants from the Natural Science Foundation of China (NSFC, No. 81371270) to XX and (No. 81271509) to QL as well as "Youth Science Talents to Sail Plan" of the Science and Technology Commission of Shanghai (14YF1400700) to HL.

Open Access This article is distributed under the terms of the Creative Commons Attribution 4.0 International License (http:// creativecommons.org/licenses/by/4.0/), which permits unrestricted use, distribution, and reproduction in any medium, provided you give appropriate credit to the original author(s) and the source, provide a link to the Creative Commons license, and indicate if changes were made.

\section{References}

Boccuto L, Lauri M, Sarasua SM, Skinner CD, Buccella D, Dwivedi A, Orteschi D, Collins JS, Zollino M, Visconti P, Dupont B, Tiziano D, Schroer RJ, Neri G, Stevenson RE, Gurrieri F, Schwartz CE (2013) Prevalence of SHANK3 variants in patients with different subtypes of autism spectrum disorders. Eur J Hum Genet 21(3):310-316

Bockers TM, Mameza MG, Kreutz MR, Bockmann J, Weise C, Buck F, Richter D, Gundelfinger ED, Kreienkamp HJ (2001) Synaptic scaffolding proteins in rat brain. Ankyrin repeats of the multidomain Shank protein family interact with the cytoskeletal protein alphafodrin. J Biol Chem 276(43):40104-40112

Brannen KC, Panzica-Kelly JM, Danberry TL, Augustine-Rauch KA (2010) Development of a zebrafish embryo teratogenicity assay and quantitative prediction model. Birth Defects Res B Dev Reprod Toxicol 89(1):66-77

Bromley RL, Mawer GE, Briggs M, Cheyne C, Clayton-Smith J, GarciaFinana M, Kneen R, Lucas SB, Shallcross R, Baker GA (2013) The prevalence of neurodevelopmental disorders in children prenatally exposed to antiepileptic drugs. J Neurol Neurosurg Psychiatry 84(6): 637-643

Christensen J, Gronborg TK, Sorensen MJ, Schendel D, Parner ET, Pedersen LH, Vestergaard M (2013) Prenatal valproate exposure and risk of autism spectrum disorders and childhood autism. JAMA 309(16):1696-1703

Collas P (1998) Modulation of plasmid DNA methylation and expression in zebrafish embryos. Nucleic Acids Res 26(19):4454-4461

Cowden J, Padnos B, Hunter D, MacPhail R, Jensen K, Padilla S (2012) Developmental exposure to valproate and ethanol alters locomotor activity and retino-tectal projection area in zebrafish embryos. Reprod Toxicol 33(2):165-173

Dufour-Rainfray D, Vourc'H P, Le Guisquet AM, Garreau L, Ternant D, Bodard S, Jaumain E, Gulhan Z, Belzung C, Andres CR, Chalon S, Guilloteau D (2010) Behavior and serotonergic disorders in rats 
exposed prenatally to valproate: a model for autism. Neurosci Lett 470(1):55-59

Durand CM, Betancur C, Boeckers TM, Bockmann J, Chaste P, Fauchereau F, Nygren G, Rastam M, Gillberg IC, Anckarsater H, Sponheim E, Goubran-Botros H, Delorme R, Chabane N, MourenSimeoni MC, de Mas P, Bieth E, Roge B, Heron D, Burglen L, Gillberg C, Leboyer M, Bourgeron T (2007) Mutations in the gene encoding the synaptic scaffolding protein SHANK3 are associated with autism spectrum disorders. Nat Genet 39(1):25-27

Durand CM, Perroy J, Loll F, Perrais D, Fagni L, Bourgeron T, Montcouquiol M, Sans N (2012) SHANK3 mutations identified in autism lead to modification of dendritic spine morphology via an actin-dependent mechanism. Mol Psychiatry 17(1):71-84

Fischer A, Sananbenesi F, Mungenast A, Tsai LH (2010) Targeting the correct HDAC(s) to treat cognitive disorders. Trends Pharmacol Sci 31(12):605-617

Gauthier J, Champagne N, Lafreniere RG, Xiong L, Spiegelman D, Brustein E, Lapointe M, Peng H, Cote M, Noreau A, Hamdan FF, Addington AM, Rapoport JL, Delisi LE, Krebs MO, Joober R, Fathalli F, Mouaffak F, Haghighi AP, Neri C, Dube MP, Samuels ME, Marineau C, Stone EA, Awadalla P, Barker PA, Carbonetto S, Drapeau P, Rouleau GA (2010) De novo mutations in the gene encoding the synaptic scaffolding protein SHANK3 in patients ascertained for schizophrenia. Proc Natl Acad Sci U S A 107(17): $7863-7868$

Gerlai R (2010) Zebrafish antipredatory responses: a future for translational research? Behav Brain Res 207(2):223-231

Gerlai R (2011) A small fish with a big future: zebrafish in behavioral neuroscience. Rev Neurosci 22(1):3-4

Geschwind DH, Levitt P (2007) Autism spectrum disorders: developmental disconnection syndromes. Curr Opin Neurobiol 17(1):103111

Grabrucker AM, Schmeisser MJ, Schoen M, Boeckers TM (2011) Postsynaptic ProSAP/Shank scaffolds in the cross-hair of synaptopathies. Trends Cell Biol 21(10):594-603

Grzadzinski R, Huerta M, Lord C (2013) DSM-5 and autism spectrum disorders (ASDs): an opportunity for identifying ASD subtypes. Mol Autism 4(1):12

Guo, P., Z. Huang, T. Tao, X. Chen, W. Zhang, Y. Zhang and C. Lin (2015). "Zebrafish as a model for studying the developmental neurotoxicity of propofol." Journal of Applied Toxicology: n/a-n/a.

Hayashi MK, Tang C, Verpelli C, Narayanan R, Stearns MH, Xu RM, Li H, Sala C, Hayashi Y (2009) The postsynaptic density proteins Homer and Shank form a polymeric network structure. Cell 137(1):159-171

Jacob J, Ribes V, Moore S, Constable SC, Sasai N, Gerety SS, Martin DJ, Sergeant CP, Wilkinson DG, Briscoe J (2014) Valproic acid silencing of ascl1b/Ascl1 results in the failure of serotonergic differentiation in a zebrafish model of fetal valproate syndrome. Disease Models \& Mechanisms 7(1):107-117

Jiang YH, Ehlers MD (2013) Modeling autism by SHANK gene mutations in mice. Neuron 78(1):8-27

Kalueff AV, Gebhardt M, Stewart AM, Cachat JM, Brimmer M, Chawla JS, Craddock C, Kyzar EJ, Roth A, Landsman S, Gaikwad S, Robinson K, Baatrup E, Tierney K, Shamchuk A, Norton W, Miller N, Nicolson T, Braubach O, Gilman CP, Pittman J, Rosemberg DB, Gerlai R, Echevarria D, Lamb E, Neuhauss SC, Weng W, Bally-Cuif L, Schneider H (2013) Towards a comprehensive catalog of zebrafish behavior 1.0 and beyond. Zebrafish 10(1): $70-86$

Kalueff AV, Stewart AM, Gerlai R (2014) Zebrafish as an emerging model for studying complex brain disorders. Trends Pharmacol Sci 35(2):63-75

Kozol RA, Cukier HN, Zou B, Mayo V, De Rubeis S, Cai G, Griswold AJ, Whitehead PL, Haines JL, Gilbert JR, Cuccaro ML, Martin ER, Baker JD, Buxbaum JD, Pericak-Vance MA, Dallman JE (2015)
Two knockdown models of the autism genes SYNGAP1 and SHANK3 in zebrafish produce similar behavioral phenotypes associated with embryonic disruptions of brain morphogenesis. Genetics, Human Molecular

Li X, Jia S, Wang S, Wang Y, Meng A (2009) Mta3-NuRD complex is a master regulator for initiation of primitive hematopoiesis in vertebrate embryos. Blood 114(27):5464-5472

Lim S, Naisbitt S, Yoon J, Hwang JI, Suh PG, Sheng M, Kim E (1999) Characterization of the Shank family of synaptic proteins. Multiple genes, alternative splicing, and differential expression in brain and development. J Biol Chem 274(41):29510-29518

McGuinness TM, Johnson K (2013) DSM-5 changes in the diagnosis of autism spectrum disorder. J Psychosoc Nurs Ment Health Serv 51(4):17-19

Milutinovic S, D’Alessio AC, Detich N, Szyf M (2006) Valproate induces widespread epigenetic reprogramming which involves demethylation of specific genes. Carcinogenesis 28(3):560-571

Pather S, Gerlai R (2009) Shuttle box learning in zebrafish (Danio rerio). Behav Brain Res 196(2):323-327

Peca J, Feliciano C, Ting JT, Wang W, Wells MF, Venkatraman TN, Lascola CD, Fu Z, Feng G (2011) Shank3 mutant mice display autistic-like behaviours and striatal dysfunction. Nature 472(7344): 437-442

Rozen S, Skaletsky H (2000) Primer3 on the WWW for general users and for biologist programmers. Methods Mol Biol 132:365-386

Schor IE, Rascovan N, Pelisch F, Allo M, Kornblihtt AR (2009) Neuronal cell depolarization induces intragenic chromatin modifications affecting NCAM alternative splicing. Proc Natl Acad Sci U S A 106(11):4325-4330

Sedgwick SG, Smerdon SJ (1999) The ankyrin repeat: a diversity of interactions on a common structural framework. Trends Biochem Sci 24(8):311-316

Selderslaghs IW, Van Rompay AR, De Coen W, Witters HE (2009) Development of a screening assay to identify teratogenic and embryotoxic chemicals using the zebrafish embryo. Reprod Toxicol 28(3):308-320

Shaw P, Greenstein D, Lerch J, Clasen L, Lenroot R, Gogtay N, Evans A, Rapoport J, Giedd J (2006) Intellectual ability and cortical development in children and adolescents. Nature 440(7084):676-679

Sheng M, Kim E (2000) The Shank family of scaffold proteins. J Cell Sci 113(Pt 11):1851-1856

Stewart AM, Cachat J, Green J, Gaikwad S, Kyzar E, Roth A, Davis A, Collins C, El-Ounsi M, Pham M, Kalueff AV (2013) Constructing the habituome for phenotype-driven zebrafish research. Behav Brain Res 236(1):110-117

Stewart AM, Nguyen M, Wong K, Poudel MK, Kalueff AV (2014) Developing zebrafish models of autism spectrum disorder (ASD). Prog Neuro-Psychopharmacol Biol Psychiatry 50:27-36

Teixido E, Pique E, Gomez-Catalan J, Llobet JM (2013) Assessment of developmental delay in the zebrafish embryo teratogenicity assay. Toxicol in Vitro 27(1):469-478

Terbach N, Shah R, Kelemen R, Klein PS, Gordienko D, Brown NA, Wilkinson CJ, Williams RS (2011) Identifying an uptake mechanism for the antiepileptic and bipolar disorder treatment valproic acid using the simple biomedical model Dictyostelium. J Cell Sci 124(Pt 13):2267-2276

Thisse C, Thisse B (2008) High-resolution in situ hybridization to wholemount zebrafish embryos. Nat Protoc 3(1):59-69

van Spronsen M, Hoogenraad CC (2010) Synapse pathology in psychiatric and neurologic disease. Curr Neurol Neurosci Rep 10(3):207214

Verpelli C, Dvoretskova E, Vicidomini C, Rossi F, Chiappalone M, Schoen M, Di Stefano B, Mantegazza R, Broccoli V, Bockers TM, Dityatev A, Sala C (2011) Importance of Shank3 protein in regulating metabotropic glutamate receptor 5 (mGluR5) expression and signaling at synapses. J Biol Chem 286(40):34839-34850 
Wang X, McCoy PA, Rodriguiz RM, Pan Y, Je HS, Roberts AC, Kim CJ, Berrios J, Colvin JS, Bousquet-Moore D, Lorenzo I, Wu G, Weinberg RJ, Ehlers MD, Philpot BD, Beaudet AL, Wetsel WC, Jiang YH (2011) Synaptic dysfunction and abnormal behaviors in mice lacking major isoforms of Shank3. Hum Mol Genet 20(15): 3093-3108

Wang X, Xu Q, Bey AL, Lee Y, Jiang YH (2014) Transcriptional and functional complexity of Shank3 provides a molecular framework to understand the phenotypic heterogeneity of SHANK3 causing autism and Shank3 mutant mice. Mol Autism 5:30

Wilson HL, Wong AC, Shaw SR, Tse WY, Stapleton GA, Phelan MC, Hu S, Marshall J, McDermid HE (2003) Molecular characterisation of the 22q13 deletion syndrome supports the role of haploinsufficiency of SHANK3/PROSAP2 in the major neurological symptoms. J Med Genet 40(8):575-584
Wood A (2014) Prenatal exposure to sodium valproate is associated with increased risk of childhood autism and autistic spectrum disorder. Evid Based Nurs 17(3):84

Yang M, Bozdagi O, Scattoni ML, Wohr M, Roullet FI, Katz AM, Abrams DN, Kalikhman D, Simon H, Woldeyohannes L, Zhang JY, Harris MJ, Saxena R, Silverman JL, Buxbaum JD, Crawley JN (2012) Reduced excitatory neurotransmission and mild autismrelevant phenotypes in adolescent Shank3 null mutant mice. J Neurosci 32(19):6525-6541

Zhu L, Wang X, Li XL, Towers A, Cao X, Wang P, Bowman R, Yang H, Goldstein J, Li YJ, Jiang YH (2014) Epigenetic dysregulation of SHANK3 in brain tissues from individuals with autism spectrum disorders. Hum Mol Genet 23(6):1563-1578 